\title{
Mechanism of Photoreorientation of Azobenzene Dyes in Molecular Films
}

\author{
Monika Schönhoff* and Michael Mertesdorf \\ Max-Planck-Institute of Colloid and Interface Science, Rudower Chaussée 5, D-12489 Berlin, Germany
}

\author{
Mathias Lösche \\ Institute of Experimental Physics I, Leipzig University, Linnéstrasse 5, D-04103 Leipzig, Germany \\ Received: July 21, 1995; In Final Form: February 10, 1996 ${ }^{\otimes}$
}

\begin{abstract}
Aminoazobenzene photoreorientation is investigated in systems in which hydrophobically substituted chromophores are organized in molecular (Langmuir-Blodgett) films on glass substrates. Whereas the cis absorption of the chromophores in $\mathrm{CHCl}_{3}$ after irradiation into their $\pi \rightarrow \pi^{*}$ absorption band is easily measured, we were not able to detect spectral changes characteristic of isomerization in the solid films during irradiation. The quantum efficiency for trans $\rightarrow$ cis isomerization in LB films is reduced at least by a factor of $\sim 10^{-4}$ from that in organic solvent. We conclude that aggregation of the chromophores in LB films limits conformational changes after photoexcitation, in distinction from the situation when aminoazobenzene derivatives are embedded in thin polymer or liquid-crystal films. Hence, the subsequent reorientation is probably driven by a mechanism that does not encompass the cis isomer. The temperature dependence of the reorientation indicates thermal activation with an activation energy $\Delta E=0.24 \mathrm{eV}$. The irradiation intensity dependence shows that the reorientation is a collective process. It is distinctly different from what would be expected if photoexcitation resulted in reorientation of individual dye molecules and is simulated in a simple model from which the intensity threshold, $I_{\mathrm{th}}=0.95 \pm 0.2 \mathrm{~mW} / \mathrm{cm}^{2}$, is retrieved. From the experimental observations we postulate that the vibronic coupling of excited states may open a radiationless deactivation channel in which the electronic excitation energy is thermalized and distributed across neighboring molecules of the excited chromophore. The excited chromophore may hence rotate by an infinitesimal amount within the steric potential of its neighbors. This process ceases only when the chromophores are rotated to an orientation in which they are no longer excited by the irradiating light beam.
\end{abstract}

\section{Introduction}

Irradiation of azobenzenes with photon energies corresponding to their electronic excitation causes changes of the orientational distribution of the transition dipole moments of the chromophores. In this process, the dipole transition moments of the chromophores evade from the polarization direction of the incident light, so that samples with an initially isotropic distribution of their molecular orientations may become anisotropic. ${ }^{1,2}$ Moreover, if such samples are irradiated under static electric fields, the transformation to macroscopically polar materials has been observed ("photoelectropoling"). ${ }^{3}$ Reorientation effects may be used for high-density information storage, ${ }^{4,5}$ and with photoassisted poling, the formation of materials with high nonlinear optical coefficients may be feasible., ${ }^{6,7}$ Further applications include the alignment of liquid-crystalline films by azobenzenes contained in a surface layer ("command surfaces"). 8,9

Photoreorientation has been observed with azobenzenes in various forms of molecular organization. In amorphous or liquid-crystalline side-chain polymers containing azobenzene derivatives the mechanism of reorientation is well understood: ${ }^{7}$ photoisomerization from trans to cis with a subsequent thermal or photoassisted isomerization back to the trans form may generally lead to a change of the orientation of an individual chromophore. After prolonged irradiation of an ensemble of chromophores, a depletion of transition moments parallel to the polarization vector of the excitation light is induced due to the orientation-selective excitation.

${ }^{\otimes}$ Abstract published in Advance ACS Abstracts, April 1, 1996.
Photoreorientation has also been observed in LangmuirBlodgett films of side-chain polymers containing azobenzenes $^{10,11}$ and in Langmuir-Blodgett films of some derivatized aminoazobenzene chromophores. ${ }^{12}$ The absorption changes on polarized or unpolarized irradiation observed in monomer films are qualitatively similar to those in polymers, and persistent polarity ( $>$ several months) can be induced by photoelectropoling with small poling fields, $\mu F<k_{B} T$ ( $\mu$ : ground state dipole moment of the chromophore; $F$ : local electric field; $k_{B}$ : Boltzmann's constant; $T$ : temperature). The direction of the induced polarity can be switched reversibly in subsequent irradiation experiments under reversed poling field directions, and poling is possible either parallel or perpendicular to the film surface. ${ }^{13,14}$

Reflection light microscopy revealed that azobenzenes in LB films are organized in micrometer-sized domains with local preferential orientations of their transition moments. Structural changes upon reorientation of the chromophores have been characterized by AFM, electron diffraction, and X-ray reflectivity. ${ }^{15}$ This showed that on the micrometer scale the film surface roughness increased on (intense) irradiation, while the chromophore interactions on a molecular length scale were found to be preserved, as indicated by the shape of the absorption spectra. These results were interpreted as local melting of the film followed by recrystallization of the molecules in a structure that is on the molecular length scale similar to that in the native film.

Meanwhile, questions persist concerning the elementary process that leads to photoreorientation of azobenzene derivative aggregates in molecular films. For these systems, experimental observations raised doubts that an isomerization process is 


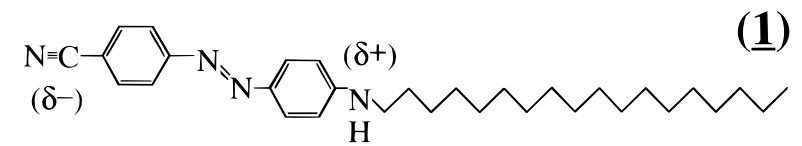

Figure 1. Chemical structure of the hydrophically substituted aminoazobenzene.

responsible for the reorientation: ${ }^{16}$ The time required for the formation of anisotropy is lower than $20 \mathrm{~ns}$ and therefore much smaller than the time constant for thermal isomerization from a putative intermediate cis isomer back to the trans conformation. In addition, the anisotropy observed after prolonged irradiation with low intensity is very small, which was attributed to a collectivity threshold of the reorientation process. ${ }^{17}$ Therefore, thermal dissipation of the excitation energy, providing a molecule with rotational mobility, was proposed as an alternate mechanism of the photoreorientation, ${ }^{16}$ and it was argued that thermally excited molecules may slightly change their orientation with respect to neighboring chromophores, so that a collective reorientation process becomes feasible. This mechanism is obviously different from trans $\rightarrow$ cis $\rightarrow$ trans isomerization, which is accepted to be responsible for photoreorientation in polymers and liquid-crystal matrixes. In both cases, however, anisotropy is induced by the orientation selectivity of the excitation process.

In this work, we try to arrive at a deeper understanding of the initial mechanism of the photoreorientation process in LB films. First, by monitoring the absorption spectra, we search for cis isomers that might be formed within the film during irradiation and analyze the absorption spectrum of the aggregated chromophores. Next, we analyze the temperature dependence of the reorientation which will provide evidence that radiationless processes other than isomerization reactions are involved in the depopulation of the excited state. Finally, the dependence of the macroscopic reorientation on the irradiation intensity is quantified and discussed in a simple model.

\section{Experimental Section}

LB films of the hydrophobically substituted aminoazobenzene (1), 4-[4'- $N$-octadecylamino]phenylazocyanobenzene (Figure 1) were prepared on glass substrates as described earlier. ${ }^{15}$ For absorption experiments, typically 10 or 20 layers of $\mathbf{1}$ were deposited by subsequent horizontal ("Langmuir-Schaefer") transfer.

The structural characteristics of these films have been investigated earlier: ${ }^{15}$ the molecules are organized in domains with diameters in the $0.1-1 \mu \mathrm{m}$ range. The film surface is rough, but locally the film consists of distinct multilayers, as indicated by a Bragg peak observed in X-ray reflectivity. As shown with atomic force microscopy and electron diffraction, the lateral organization of the molecules at the film surface is an aggregate structure of crystalline order with a coherence length of $>250 \AA .^{18}$

Routinely, absorption measurements were performed in a standard dual-beam UV/vis spectrometer (Perkin-Elmer Lambda 17; setup A). For measurements of small absorption changes, experiments were conducted in a single-beam spectrometer of local design (setup B), ${ }^{13}$ where the sample can be rotated by $90^{\circ}$ into the irradiation beam without dismounting, thereby avoiding artifacts due to changes in the sample position. For photoreorientation, samples were irradiated with an $\mathrm{Ar}^{+}$ion laser $(\lambda=488 \mathrm{~nm})$. To ensure uniform light intensity across the irradiated area, the laser beam was widened and a diaphragm was used to cut off the areas with an intensity drop of more

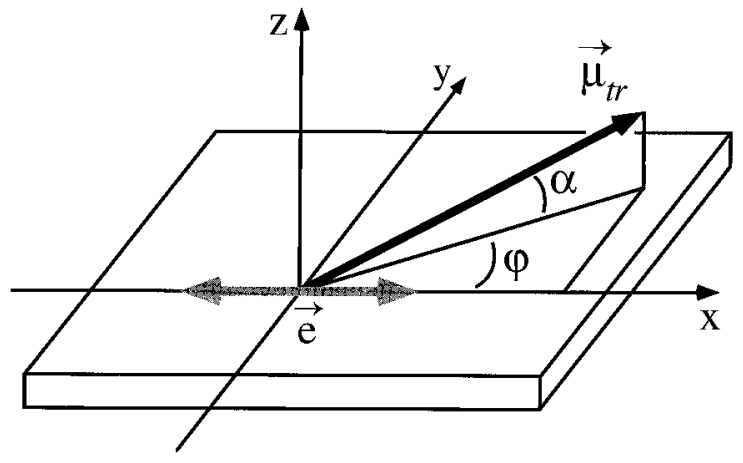

Figure 2. Geometry of irradiation and definition of the angles $\alpha$ and $\varphi$ : the light beam is incident parallel to $\vec{z}$ and the film is in the $\vec{x}-\vec{y}$ plane. $\vec{e}$ : polarization vector of the irradiation; $\vec{\mu}_{\mathrm{tr}}$ : transition dipole moment.

than $10 \%$ below the maximum intensity in the beam center. Irradiation times were typically 5 or $10 \mathrm{~min}$ with linearly polarized light. For absorption measurements during crossirradiation (setup C), an optical multichannel analyzer (OSMA, Spectroscopy Instruments) was used as a detector to quantify the light transmitted through the sample. From the transmission spectra sampled over $33 \mathrm{~s}$, the absorption of the molecular films was computed. Spectrometer and laser beams were both incident under $45^{\circ}$ on the sample and perpendicular with respect to each other in this configuration.

In our home-built spectrometer, the sample was optionally cooled by means of a Joule-Thomson type He cryostat (Cryogenics, Model 22), in which the inner sample chamber was filled with $\mathrm{He}$ as a contact gas. Due to the geometry of the cryostat, it was not possible to perform absorption measurements and irradiation on precisely the same sample area. Therefore, the transmission of the sample at low temperature prior to and after irradiation was measured without using the spectrometer by simply monitoring the laser intensity after the sample with a photodiode (setup D).

Figure 2 illustrates the sample geometry for irradiation and all subsequent absorption measurements (except setup C): the $x-y$ plane is the plane of the surface film, and the incident light beam (for irradiation or measurement of the transmission) is parallel to the $\vec{z}$ axis. The laser beam is linearly polarized with its electric field vector, $\vec{e}$, parallel to $\vec{x}$. The orientation of a molecule is described by the angles $\alpha$ and $\varphi$ as indicated by the fat arrow that represents the long chromophore axis, which nearly coincides with the direction of the electric transition dipole moment, $\vec{\mu}$, of the $\pi \rightarrow \pi^{*}$ excitation.

\section{Results}

I. Isomerization. To investigate whether or not photoisomerization is involved in the reorientation process, the lightdependent absorption spectra of compound $\mathbf{1}$ in $\mathrm{CHCl}_{3}$ solution have been determined. Figure $3 \mathrm{a}$ shows the absorption before irradiation and at different times after the end of an irradiation at $\lambda=488 \mathrm{~nm}$. Before irradiation, only one absorption band with a maximum at $\lambda=429 \mathrm{~nm}$ is observed. It corresponds to the $\pi \rightarrow \pi^{*}$ transition of the trans isomer. The shoulder at $\lambda=$ $360 \mathrm{~nm}$ observed upon laser irradiation can be assigned to the $\pi \rightarrow \pi^{*}$ transition of the cis isomer. Corresponding to the increase of the cis concentration, the trans concentration decreases with irradiation. After shutoff of the laser beam, monoexponential thermal relaxation of the cis population back to equilibrium was observed with $\tau_{\text {cis }}=560 \pm 20 \mathrm{~s}$; see inset in Figure 3a. For a similar azobenzene derivative, 4-(hexylamino)- $4^{\prime}$-nitroazobenzene, time constants of the same order of 

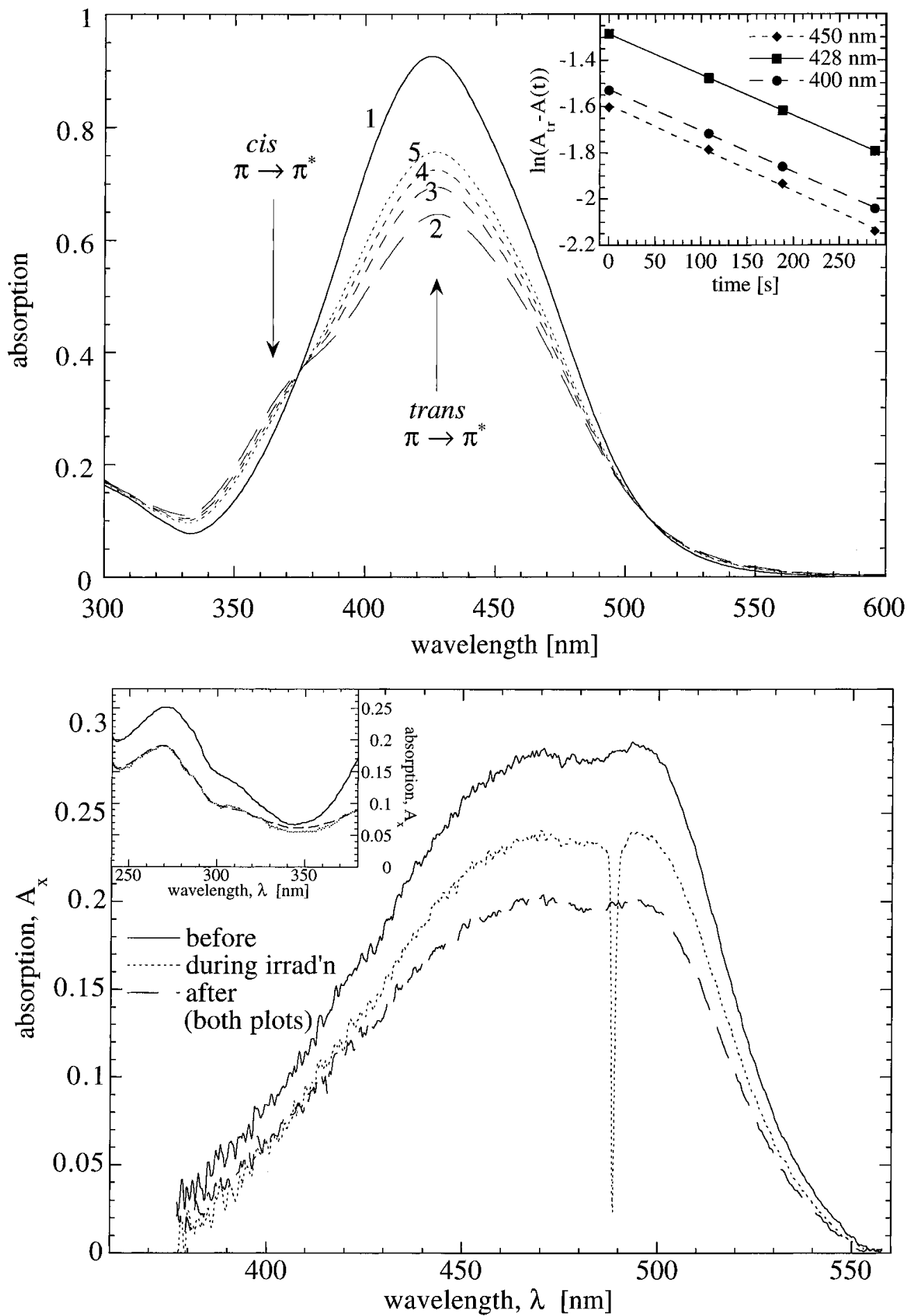

Figure 3. (a, top) Absorption spectra of $0.15 \mathrm{mmol} / \mathrm{L}$ of 1 in $\mathrm{CHCl}_{3}$. Trace 1: prior to irradiation, trace 2: $0 \mathrm{~s}$; trace 3: $108 \mathrm{~s}$; trace 4: $188 \mathrm{~s}$; trace 5: $289 \mathrm{~s}$ after switching off the irradiation. Inset: graphic evaluation of the decay at different wavelengths as indicated. (b, bottom) Absorption spectra of 10 layers of $\mathbf{1}$ deposited on a glass slide, measured with $x$-polarized light prior to, during and after irradiation. The drop at $488 \mathrm{~nm}$ is due to laser stray light. The near-UV region shown in the inset is not background corrected.

magnitude have been reported in nonpolar solvents $\left(\tau_{\text {cis }}=500\right.$ $\mathrm{s}),{ }^{19}$ whereas in polar solvents ${ }^{19}$ and in polymers ${ }^{20,21}$ the relaxation may be up to 2 orders of magnitude faster.

Corresponding experiments on compound 1 in LB films show that on deposition to solid substrates, the $\pi \rightarrow \pi^{*}$ transition shifts from $\lambda=429 \mathrm{~nm}$ and splits, presumably due to aggregation of the chromophores, into two distinct bands at $\lambda=460 \mathrm{~nm}$ and $\lambda=495 \mathrm{~nm}$. We will refer to them hereafter as aggregate bands. However, such experiments did not reveal a change of the absorption spectrum upon irradiation. To ascertain that we did not miss a short-lived cis population, we took absorption measurements during irradiation every $33 \mathrm{~s}$ with the OSMA spectrometer (setup C). Figure $3 b$ shows spectra of the $\vec{x}$-polarized absorption before, during and after $\vec{x}$-polarized irradiation. While the decrease of the overall absorption is mainly caused by the reorientation process, no changes of the spectral shape are observed. To access the near-UV range of the absorption, similar experiments were conducted in the UV/ vis spectrometer (setup A), with the sample turned by $45^{\circ}$ and polarized laser irradiation incident perpendicular to the probing 


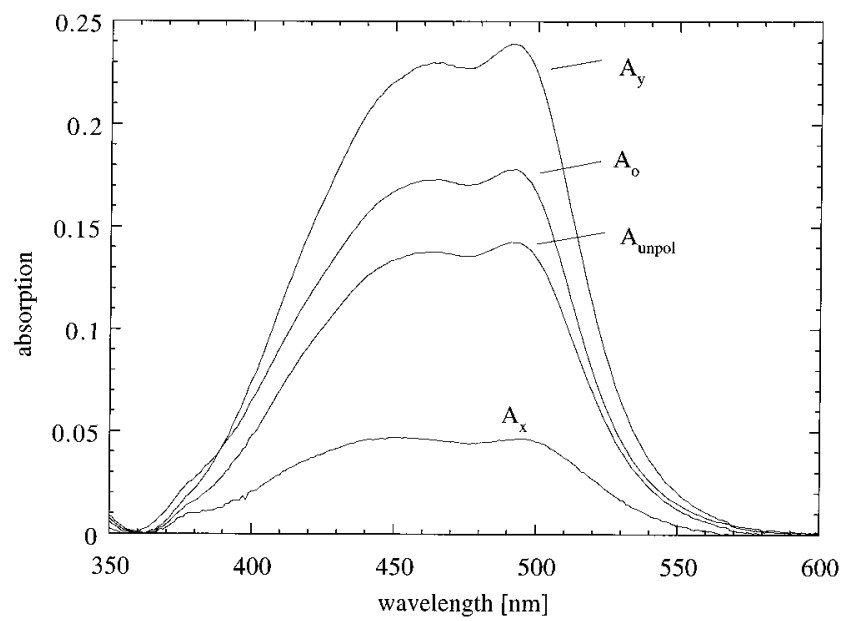

Figure 4. Absorption spectra of 6 layers of $\mathbf{1}$ on a glass substrate before $\left(A_{0}\right)$ and after irradiation $\left(\lambda=488 \mathrm{~nm}, \vec{x}\right.$ polarized): $A_{x}: \vec{x}$ polarized, $A_{y}: \vec{y}$-polarized; $A_{\text {unpol }}$ : unpolarized after irradiation.

light. The spectra shown in the inset in Figure $3 \mathrm{~b}$ are not background corrected; like the spectra shown in the main panel, they do not exhibit any changes of the spectral shape due to irradiation.

II. Polarized Irradiation. The effect of polarized irradiation on the absorption spectrum is shown in Figure 4. Prior to irradiation, the absorption is isotropic $\left(A_{o}=A_{x}=A_{y}\right)$; after irradiation, the $\vec{x}$-polarized absorption has decreased and the $\vec{y}$ polarized absorption has increased. This indicates an evasion of the transition dipole moments, and hence of the molecular axes, from the direction of $\vec{e}$, which reduces the probability of absorption of $\vec{x}$-polarized light. After irradiation, the unpolarized absorption $\left(A_{\text {unpol }}\right)$ has also significantly decreased. This is not due to photodestruction but indicates partial reorientation of the chromophores into the $\vec{z}$-direction, ${ }^{18}$ leading to an increase of $\alpha$ (cf Figure 2), and hence to a reduction of $\vec{\mu}_{\mathrm{tr} * \vec{e}}$.

Although the optical density becomes anisotropic after photoreorientation, the shape of the absorption spectra remains almost unaffected by the irradiation, showing that the two observed bands are not polarized perpendicular to each other. This rules out that both bands originate from one type of aggregate: the absorption spectrum of an aggregate that contains two molecules per unit cell is expected to split into two bands. If the molecules are not oriented parallel or antiparallel, then both transitions are allowed and are polarized perpendicularly to each other. ${ }^{22}$ On photoreorientation, these polarizations should develop differently in the polarized absorption spectra. Since no such differences are observed in the experimental spectra, the two bands cannot arise from the same aggregate. As both bands are red-shifted with respect to the solution wavelength, they may arise from two distinct types of $\mathbf{J}$ aggregates, different in their molecular packing densities. The susceptibility for photoreorientation is only marginally larger for the aggregate with its absorption maximum at $\lambda=495 \mathrm{~nm}$. This may be connected to the fact that the irradiation wavelength $(\lambda=488 \mathrm{~nm})$ is closer to the absorption maximum of this aggregate. Since this effect is only of the order of a few percent, it is neglected in the following discussion.

III. Temperature Dependence of the Reorientation. Since the nonradiative decay of the excitation energy has been proposed to be involved in the early stage of the reorientation, the relevant mechanism might leave its fingerprint in the temperature dependence of the process. As a measure of the anisotropy, the relative change of the $\vec{x}$-polarized normalized absorption was calculated from transmission measurements:

$$
1-a_{x}=\frac{A_{x}^{\circ}-A_{x}}{A_{x}^{\circ}}=-\frac{1}{A_{x}^{\circ}} \log \frac{T_{x}^{\circ}}{T_{x}}
$$

$A_{x}$ : absorption; $T_{x}$ : transmission parallel to $\vec{x}$; ${ }^{\circ}$ : before irradiation.

Figure 5 shows the experimental result: while below $T=$ $150 \mathrm{~K}$ no reorientation was observed, the onset of reorientation at about $T=220 \mathrm{~K}$ is very steep. Although a transition between phases with different rotational diffusion coefficients might cause the observed behavior, such a phase transition seems unlikely since the onset of photoreorientation occurs over an interval, $\Delta T \sim 40 \mathrm{~K}$, that is much wider than a phase transition $(\Delta T<$ several kelvin). More likely, the increase in rotational mobility is thermally activated, a possibility which will be investigated below. A decrease of $\left(1-a_{x}\right)$ is observed as $T$ approaches room temperature, such that the photoinduced anisotropy shows a maximum around $T \sim 250 \mathrm{~K}$. This is probably connected to thermal randomization of the reoriented states on increasing the rotational mobility of the chromophores further at higher temperature.

IV. Intensity Dependence of the Reorientation. To characterize the intensity threshold of the reorientation process, samples were irradiated with different intensities on different areas (setup B). First, a series of experiments was conducted with different intensities $I$ delivered on the sample in different time intervals, such that the deposited energy density, $\rho=I t$, was constant in all experiments. In a second set of experiments, $I$ was varied by varying $\rho$ at constant $t$. After irradiation, the $\vec{x}$ and $\vec{y}$ polarized absorption spectra, $A_{i}(i=x, y)$, were measured. To avoid artifacts induced by local inhomogeneities in dye concentration, all measured absorption values were normalized to the unpolarized absorption before irradiation, $A_{i}^{\circ}$, and the results, $a_{i}=A_{i} / A_{i}^{\circ}$, were plotted versus $I$.

If the reorientation of individual molecules occurs independently of each other, as it is presumably the case for azobenzene derivatives in amorphous matrices, the anisotropies obtained with constant $\rho$ should be independent of $I$ : the anisotropy will be a function of the total number of absorbed photons, regardless of their sequence in time, and only at high intensities, saturation will cause a reduction of the reorientation efficiency. In contrast to this expectation, a marked intensity dependence is observed in the first series of experiments, as depicted in Figure 6a: the anisotropy is much lower at small intensities than at high intensities, indicating that not the total number of incident photons is the important quantity but that coincidence of photons is essential for reorientation. This indicates a collective process, where the reorientation of a molecule depends strongly on the excitation probability of neighboring chromophores.

The same conclusion was derived from the second set of experiments: Figure $6 \mathrm{~b}$ shows a sigmoidal intensity dependence of the reorientation on $I$, i.e., an increasing slope of the function $a_{y}$ vs $I$ and a decreasing slope of $a_{x}$ vs $I$ close to $I=0$. The data indicate an intensity threshold of about $1 \mathrm{~mW} / \mathrm{cm}^{2}$, which is consistent with the observation of an increase of the reorientation efficiency below $I \sim 1 \mathrm{~mW} / \mathrm{cm}^{2}$ reported in Figure 6a. In particular, such a result is not expected for the reorientation of independent molecules, where the anisotropy should increase linearly with irradiation intensity at low $I$.

\section{Discussion}

We have interpreted the dependence of the photoinduced anisotropy on the incident intensity (Figure 6) as a collective process that results in reorientation of the chromophores. In such a process, the molecules have to overcome steric hindrances within their domain. One may speculate that mechanical stress 


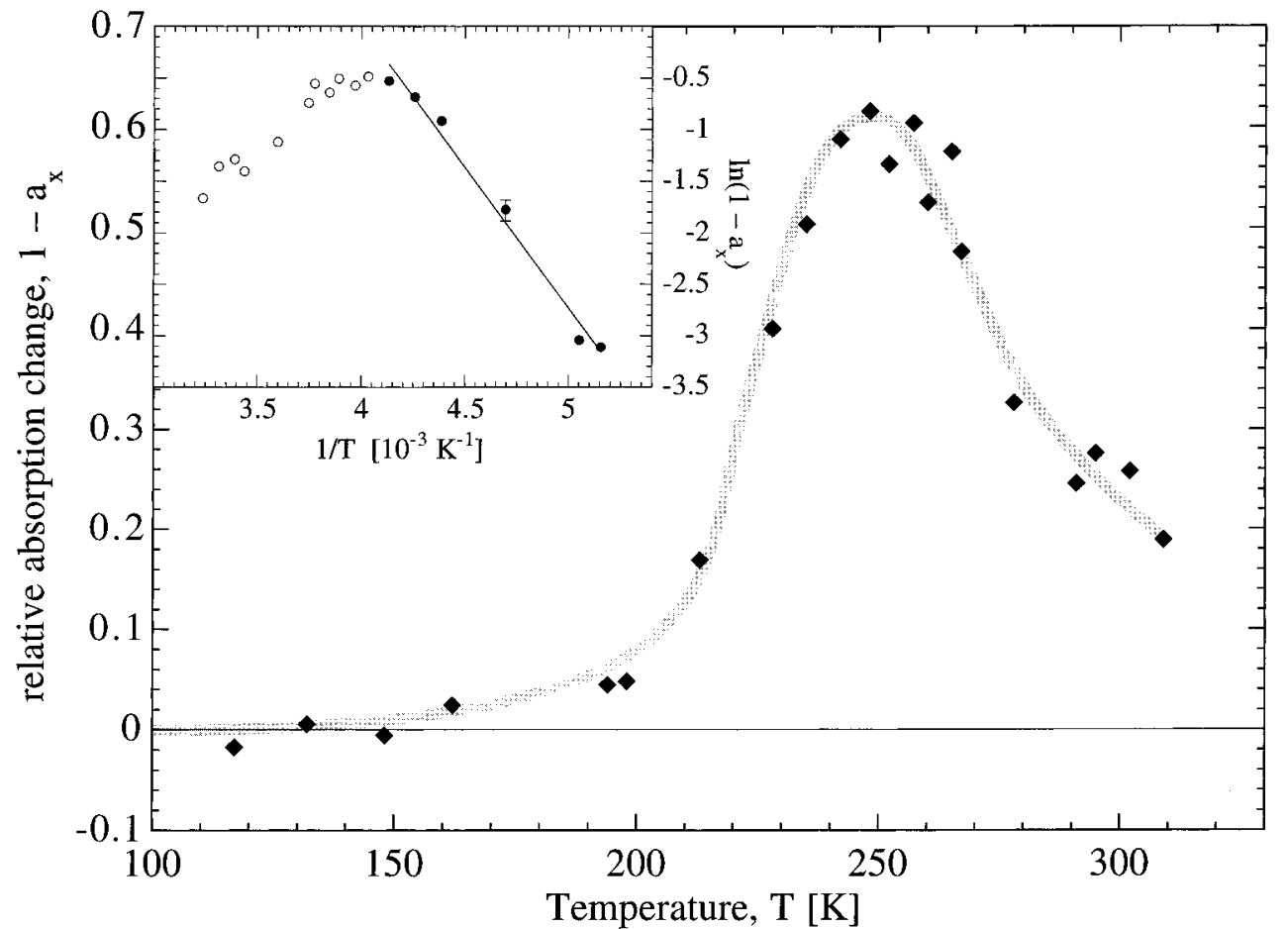

Figure 5. Relative change of in-plane anisotropy of the films after irradiation $\left(\lambda=488 \mathrm{~nm}, t=10 \min , I=3.5 \mathrm{~mW} / \mathrm{cm}^{2}\right)$, measured as $\left(1-a_{x}\right)$ as a function of temperature using setup D. The gray curve is a guide for the eye. Inset: Arrhenius plot of $\left(1-a_{x}\right)$. The slope between $T=190$ and $240 \mathrm{~K}$ (data represented by full circles) indicates an activated process with $\Delta E=0.24 \mathrm{eV}$.

is released by breaking domains into smaller subdomains. Indeed, significant structural changes on reorientation have been observed with the AFM on a length scale of some micrometers, ${ }^{15}$ and results from electron diffraction hint at the formation of even smaller domains on a scale of $250 \AA<d<1 \mu \mathrm{m} .{ }^{18}$

For a discussion of the molecular mechanism driving photoreorientation, the lack of change in the UV/vis absorption band upon irradiation (Figure 3b,c) might not seem sufficient to argue that chromophores do not exist in the cis conformation in any intermediate state during the experiment. In the following we discuss alternate possibilities why the shape of the absorption spectra might fail to change during irradiation.

The cis absorption observed in solution in the UV region might be red-shifted in the film, so that it is not detectable due to coincidence with the large trans $\pi \rightarrow \pi^{*}$ absorption band. This would imply, however, that the absorption maximum of the cis $\pi \rightarrow \pi^{*}$ transition in the deposited film is shifted by about 100 $\mathrm{nm}$ from the absorption wavelength in $\mathrm{CHCl}_{3}$ solution, where the absorption maximum occurs at $\lambda=360 \mathrm{~nm}$ (cf. Figure $3 \mathrm{a}$ ). There is no evident mechanism that might be responsible for such a large shift because, in distinction from the situation with trans isomers, there is no aggregation between chromophores in the cis form.

If it is accepted that the trans and cis $\pi \rightarrow \pi^{*}$ absorption maxima of the chromophores do not coincide in the deposited films, an upper limit of the trans $\rightarrow$ cis isomerization quantum efficiency, $\phi_{\mathrm{tr}}$, can be estimated from the results shown in Figure $3 \mathrm{~b}$ by

$$
\rho_{\text {cis }}=j_{\text {ph }} \tau_{\text {cis }} \phi_{\text {tr }}
$$

$j_{p h}$ : photon flux density per unit area; $\tau_{\text {cis }}$ : lifetime of the cis state; $\rho_{\text {cis }}$ : density of chromophores in the cis state per unit area. $\tau_{\text {cis }}$ in the deposited films is unknown a priori, but for the following estimate we will assume that it is the same as in $\mathrm{CHCl}_{3}$ solution, as was found for a different azobenzene derivative in a monolayer. ${ }^{23}$ Assuming further that a change in the spectra would be detectable if at least $10 \%$ of the chromophores were in the cis conformation in the steady state, it follows from eq 2 that

$$
\phi_{\mathrm{tr}} \leqslant 7.4 \times 10^{-6}
$$

This is orders of magnitude lower than $\phi_{\text {tr }}$ values found in the literature for similar compounds in solution, e.g., $\phi_{\mathrm{tr}}=0.03$ for 4-(dimethylamino)-4'-nitroazobenzene. ${ }^{24}$ We note that the upper limit of $\phi_{\text {tr }}$ given in eq 3 is a conservative estimate with respect to the assumption on $\tau_{\text {cis }}$, as the cis $\rightarrow$ trans isomerization is a thermally activated process. Steric hindrance between the aggregated molecules will tend to increase, rather than decrease, the activation barrier between the cis and trans states in the film, and thus $\tau_{\text {cis }}^{\text {solid film }}>\tau_{\text {cis }}^{\text {solution }}$ might be expected. Consequently, $\phi_{\text {tr }}$ must be even lower than the value given above. This means that isomerization is efficiently suppressed by the dense molecular packing of the chromophores ${ }^{15}$ in the aggregates within the deposited films.

There have been reports, however, of a fast component of the thermal relaxation cis $\rightarrow$ trans of azobenzene compounds in polymer matrixes where the lifetimes are reduced by $\sim 2$ orders of magnitude compared to solution. ${ }^{20,21}$ This reduction has been attributed to strained conformations of the cis isomers. If a similar situation occurred in LB films, $\phi_{\text {tr }}$ might be a factor of 100 larger than the upper value given in the estimate (eq 3 ). Similarly, if photoisomerization from cis back to trans is taken into account, the true value of $\tau_{\text {cis }}$ in the films might be further reduced and that of $\phi_{\text {tr }}$ further increased. Such a contribution, however, is again expected to be small, if one puts into a quantification the quantum efficiencies and optical densities determined for a compound similar to $1,{ }^{20}$ and cannot bring $\phi_{\text {tr }}$ up to its solution value.

The most probable scenario is thus that local spatial constrictions reduce the free volume of the molecules such that major changes in their configuration like trans $\rightarrow$ cis isomerization is no longer possible. The importance of the free volume has been 

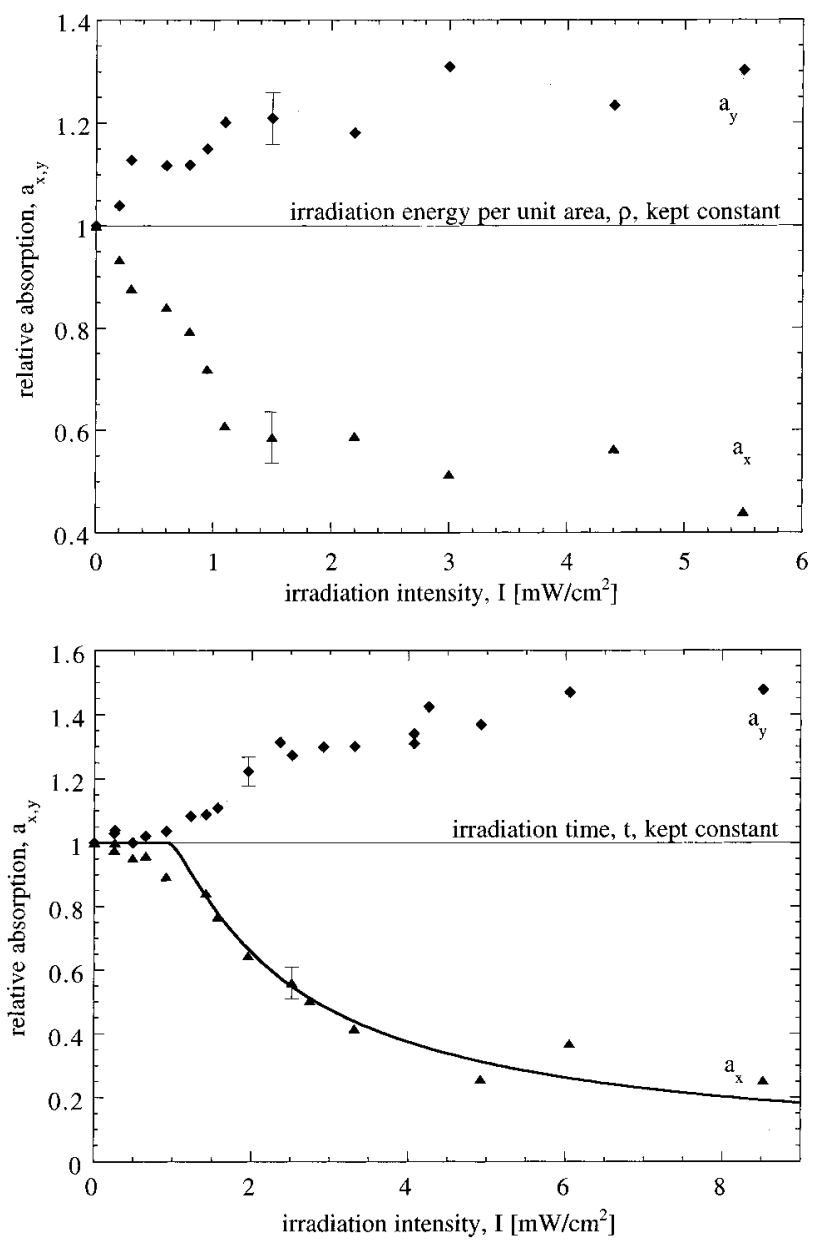

Figure 6. Relative polarized absorption $a_{x, y}=A_{x, y}$ (after irradiation)/ $A_{x, y}$ (before irradiation), calculated from the absorption maximum at $\lambda$ $=492 \mathrm{~nm}$. (a) Irradiation intensity $I$ and the irradiation time $t$ were varied such that the energy density $\rho$ remained constant. (b) Intensity $I$ and thereby the irradiation energy $\rho$ were varied while keeping the irradiation time $t$ constant. Gray curves are guides for the eye. The solid curve in the lower part of $\mathrm{b}$ is a fit with the model represented by eq 6 .

demonstrated for azobenzene derivatives similar to $1 .^{25} \mathrm{~A}$ suppression of the isomerization in monolayers on the water surface has been observed with increasing surface pressure. Also, in transferred layers of azobenzene derivatives, the quantum efficiency of isomerization has been shown to be reduced by aggregation. ${ }^{26}$ For the systems investigated here, we conclude that there is no significant photostationary concentration of the cis state. Reorientation is brought about by a mechanism other than isomerization. A short-lived population of strained cis isomers that could play a minor role in this process may on the basis of the experimental data not entirely excluded. In any case it is worth while noting, that isomerization has not necessarily to be involved: photoreorientation has been reported of a diacetylene compound that is not capable of isomerization reactions. ${ }^{27}$

Since photoexcitation is required for reorientation but the trans $\rightarrow$ cis isomerization in deposited films is restricted by steric hindrance between neighboring molecules within molecular aggregates, another deactivation mechanism of the electronic excitation energy may be relevant for photoreorientation. Figure 7 shows a schematic potential energy diagram of (unsubstituted) azobenzene as given by Rau. ${ }^{28}$ Indicated are several deactivation pathways which may or may not involve the cis conformation of the molecule. Cis may be formed from the trans state via two different mechanisms: rotation of the two half-

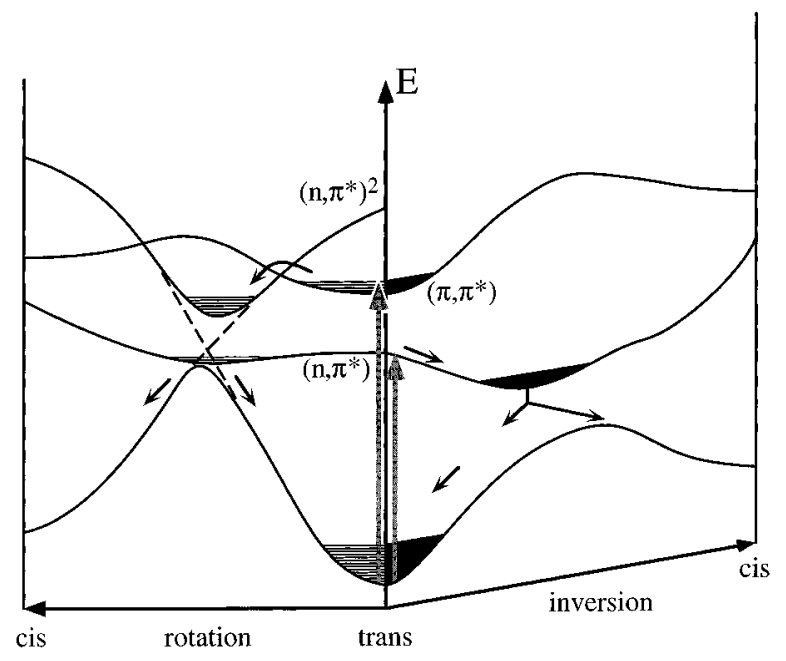

Figure 7. Schematic potential energy diagram of unsubstituted azobenzene according to Rau. ${ }^{24}$ The electronic states are shown in dependence on the molecular coordinates that are relevant for isomerization. The arrows indicate possible reaction paths after excitation of the chromophore in the trans configuration.

molecules against each other around the azo bond after transient breakage of the double bond or inversion via a planar transition state. $^{24}$ Both possibilities are accounted for in Figure 7 by plotting two independent degrees of freedom that connect trans and cis, so that the conformations on the far left and far right positions of the diagram are identical. Possible deactivation pathways after $n \rightarrow \pi^{*}$ or $\pi \rightarrow \pi^{*}$ excitation are indicated encompassing radiationless transitions to the trans or cis ground states. For amino substituted azobenzenes, the energy of the $\pi \rightarrow \pi^{*}$ transition, which is the dominant electronic absorption band, is lowered compared to the situation shown in Figure 7 and overlaps with the $n \rightarrow \pi^{*}$ transition.

Restriction of the molecular conformations in the aggregates dictates that relaxation has to take place within a limited range around the trans configuration, and the overlap of the $\pi \rightarrow \pi^{*}$ and the $n \rightarrow \pi^{*}$ transitions might enhance the probability of such conformationally restricted relaxation. This is hinted at by the observation of photoreorientation for a number of different aminoazobenzenes, but a complete absence of reorientation in LB films of oxoazobenzenes, ${ }^{29}$ which lack the degeneracy of $\left(\pi \pi^{*}\right)$ and $\left(\mathrm{n} \pi^{*}\right)$. This observation would suggest that depopulation of $\left(\pi \pi^{*}\right)$ proceeds via $\left(\mathrm{n} \pi^{*}\right)$ with minute shifts in atomic coordinates for aminoazobenzenes, a mechanism which may be disabled for other azobenzene derivatives. In the conformationally restricted relaxation process, the electronic excitation energy is converted to thermal energy, which increases slightly the rotational mobility of the molecule in the steric potential of its neighbors within the aggregate. The thermal energy is subsequently dissipated over the molecular aggregate. For more definite conclusions about the relaxation path, however, a precise knowledge of the potential energy diagram of $\mathbf{1}$ is essential.

To understand the temperature dependence of the reorientation efficiency (cf. Figure 5), an Arrhenius plot of the experimental results is given in an inset in Figure 5. For an interpretation of this plot, the relative change $\left(1-a_{x}\right)$ of the polarized absorption is taken as a measure of reorientation, since $a_{x}$ comprises a macroscopic average over the local orientations. A linear dependence is found in the region between $T=190$ and 240 $\mathrm{K}$, consistent with

$$
\left(1-a_{x}\right)=c \exp \left(-\Delta E / k_{\mathrm{B}} T\right)
$$

The activation energy is $\Delta E=0.24 \mathrm{eV}$, typical for a vibrational 
process with $\tilde{v}=1900 \mathrm{~cm}^{-1}$. This result suggests that for reorientation not only electronic excitation but also thermal population of vibrational levels may be essential. In the frame of the schematic potential diagram discussed above (cf. Figure 7 ), it is crucial to reach the intersection of two states for radiationless deactivation to occur, and a thermally excited molecular vibration might provide the necessary energy. In this picture, the activation behavior of the temperature dependence with a typical oscillation energy can be understood: an intramolecular vibration mode may couple to the electronic excitation, and only by the excitation of this vibration intramolecular coordinates are reached, from which radiationless transitions occur. While $\Delta E$ corresponds well to typical vibrational energies, we find $k_{B} T \ll \Delta E$, implying that a very low occupancy, $\sim 10^{-5}$, of the excited vibrational level is sufficient to account for efficient radiationless deactivation.

The main conclusion from the experimental facts presented above is thus that the driving mechanism for photoreorientation of $\mathbf{1}$ in deposited molecular films is most likely a dissipation of the electronic excitation energy via vibronic channels and that trans $\rightarrow$ cis $\rightarrow$ trans isomerization plays a minor role, if any. This is markedly different from the situation where azobenzene derivatives are dissolved in polymer or liquid-crystalline matrixes. In all cases, photoselection leads to an excitation of those chromophores with a major component of their transition moments parallel to the polarization of the incident light. With aminoazobenzenes in deposited films, the thermalization of the excitation energy may locally increase the rotational mobility of the photoselected chromophores that are confined within tightly packed molecular aggregates. Within these domains, the transition moments are collectively driven out of the light polarization: a slight rotation of a chromophore after photoexcitation toward the polarization direction will enhance the probability for its reexcitation; the opposite is true if the chromophore happens to be driven toward an orientation perpendicular to the polarization. The orientational distribution of the chromophores within the domain is thus deformed in infinitesimal steps, stabilized by the collectivity of the process, to yield a shift of the orientational distribution away from the polarization vector of the incident light.

The decrease of the orientational anisotropy observed for photoreorientation at $T>250 \mathrm{~K}$ may be due to an increase of the width of the orientational distribution within a domain or to an enhancement of temporal fluctuations by thermal energy.

\section{Model for Collective Reorientation}

For a quantitative interpretation of the intensity dependence of the reorientation, it has to be taken into account that the observed polarized absorption represents a macroscopic average over many domains that differ in their director orientations. The averaged excitation probability of the chromophores within a domain depends on the director orientation: $p_{e x c} \propto\left(\vec{e}_{t r}\right)^{2}$. This can approximately be described as an "effective" irradiation intensity, $I_{\text {eff }}=I \cos ^{2} \varphi$, where $\varphi$ is the azimuthal angle of the director with respect to $\vec{e}$ (cf. Figure 2).

To reproduce the impact of averaging the optical absorption over a macroscopic area of a film, a model for the reorientation of a single domain was implemented, in which a subsequent integration over the whole ensemble of domains was performed: a domain is characterized by the orientation of its director and the width of the orientational distribution is neglected. A local intensity threshold $I_{\text {th }}$ was introduced, below which the director rotation was assumed to cease. The intensity threshold is taken to be rigorous with reorientation of the domain taking place only when the effective intensity $I_{\text {eff }}$ at the domain exceeds $I_{\text {th }}$. Therefore, a domain with a given initial orientation $\varphi$, will reorient from the direction of $\vec{e}$ until $I_{\mathrm{eff}}=I_{\mathrm{th}}$, resulting in a director orientation $\varphi_{\text {th }}$ connected to $I_{\text {th }}$ by $I_{\text {th }}=I \cos ^{2} \varphi_{\text {th }}$. In the model it is assumed that the domains are isotropically distributed prior to reorientation $\left(f_{0}(\varphi)=1 /(2 \pi)\right)$. The measured spectra show a migration not only inplane but also toward the $z$ axis, which will be neglected in the model. The final orientation distribution $f_{\text {final }}(\varphi)$ of the domain directors after irradiation will then consist of a fraction of domains that have not been affected by irradiation, since their initial orientations $\varphi_{0}$ were too far from the direction of $\vec{e}$, and of a reoriented fraction of domains that have been driven into a final director orientation at $\varphi=\varphi_{\mathrm{th}}$ :

$$
f_{\text {final }}(\varphi)= \begin{cases}c * \delta\left(\varphi-\varphi_{\mathrm{th}}\right) & \left(\varphi_{0} \leq \varphi_{\mathrm{th}}\right) \\ 1 /(2 \pi) & \left(\varphi_{0}>\varphi_{\mathrm{th}}\right)\end{cases}
$$

$c=c\left(\varphi_{\mathrm{th}}\right)=\varphi_{\mathrm{th}} /(2 \pi)$ : normalization constant; $\delta$ : Dirac function. This distribution function $f_{\text {final }}(\varphi)$ is used to calculate the polarized absorption. The result is

$$
\begin{aligned}
& A_{x}=\frac{2}{\pi} A_{\mu}\left[\frac{\pi}{4}-\frac{\varphi_{\mathrm{th}}}{2}-\frac{1}{4} \sin \left(2 \varphi_{\mathrm{th}}\right)+\varphi_{\mathrm{th}} \cos ^{2} \varphi_{\mathrm{th}}\right] \\
& A_{y}=\frac{2}{\pi} A_{\mu}\left[\frac{\pi}{4}-\frac{\varphi_{\mathrm{th}}}{2}-\frac{1}{4} \sin \left(2 \varphi_{\mathrm{th}}\right)+\varphi_{\mathrm{th}} \sin ^{2} \varphi_{\mathrm{th}}\right]
\end{aligned}
$$

$A_{\mu}$ : absorption for transition moments parallel to $\vec{e}$.

The dependence of $A_{x}$ on $I$ is fitted to the experimental data shown in Figure $6 \mathrm{~b}$, with $\varphi_{\text {th }}$ (and hence implicitly $I_{\text {th }}$ ) as the sole independent parameter. The result is given as a solid line in Figure $6 \mathrm{~b}$ and shows that the experimental data are well described with $I_{\text {th }}=0.95 \pm 0.2 \mathrm{~mW} / \mathrm{cm}^{2}$. The deviation between data and model in the region of $I \sim I_{t h}$ may be due to the assumption of an ideally sharp threshold. Releasing this condition would result in a smearing of the macroscopic model curve. The neglect of partial reorientation toward the surface normal does not affect $A_{x}$ but it does affect $A_{y}$ : the intensity dependence of $A_{y}$ does not correspond as well to the experimental data as that of $A_{x}$, since the reorientation toward $\vec{z}$ causes a smaller increase of $A_{y}$ with $I$ than anticipated in the model.

The simulation results show that the measured intensity dependence of the anisotropy is well accounted for by a simple concept if macroscopic averaging is applied. The model describes much better the experiments, in which the irradiation time was kept constant, than those with constant energy density. This is related to the model assumption that the reorientation is complete (after a "constant" irradiation time $t=$ " $\infty$ "). Therefore, the velocity of the director rotation, which may be intensity dependent in the experiments, does not enter into the results from the model.

Intensity thresholds for the reorientation of $\mathbf{1}$ in deposited films has already been reported earlier. ${ }^{24}$ It was shown that reorientation occurs above $I_{\mathrm{th}}^{\mathrm{cw}} \sim 3 \mathrm{~mW} / \mathrm{cm}^{2}$ on broadband $\mathrm{cw}$ irradiation or at energy densities above $\epsilon_{\mathrm{th}}^{\mathrm{p}} \sim 5 \times 10^{-2} \mathrm{~J} / \mathrm{cm}^{2}$ on pulsed laser irradiation $(\Delta t \sim 20 \mathrm{~ns}$ at $\lambda=532 \mathrm{~nm})$. While the $\mathrm{cw}$ intensity threshold is comparable to that reported here (the difference, a factor of 3 , is consistent with a reduction of sensitivity of the chromophores to the broadband spectrum of the irradiation used in the earlier work), there is a large discrepancy to the pulsed beam experiment, where $I_{\mathrm{th}}^{\mathrm{p}}=$ $\epsilon_{\mathrm{th}}^{\mathrm{p}} / \Delta t \sim 2.5 \times 10^{6} \mathrm{~W} / \mathrm{cm}^{2}$ is orders of magnitude higher than in the cw experiments. This huge difference in efficiency indicates that the characteristic time for the reorientation process is much longer than the pulse duration $\Delta t$. The process is 
unlikely to involve single chromophores, such as an isomerization would, because then the characteristic time would probably be faster than the laser pulse. Again, we conclude, that a slow process, such as the distribution of thermal energy over the molecules within a solid domain, that is following the fast thermal deactivation, is the driving force for photoreorientation.

From the cw threshold intensity $I_{\text {th }}$, the minimum density of electronically excited molecules $\rho^{*}$ in the film that is necessary to support photoreorientation, can be computed:

$$
\rho^{*}=\frac{I_{\mathrm{th}}}{h v} \tau^{*} p_{\mathrm{A}}
$$

$\tau^{*}$ : lifetime of the excitation; $p_{A}$ : absorption probability). Expressing $\rho^{*}$ by the proportion $x^{*}$ of excited molecules in the photostationary state and by the total number density $\rho_{\text {total }}$ of chromophores per unit area in the film, $x^{*}=\rho^{*} / \rho_{\text {total }}$, would yield

$$
x^{*}=3 \times 10^{-10}
$$

if $\tau^{*}=1 \mathrm{~ns}$ is used as characteristic time for a molecular process $\left(p_{A} \sim 0.06\right.$ is taken from the absorbance of a monomolecular film and $\rho_{\text {total }} \sim 5 \times 10^{14} \mathrm{~cm}^{-2}$ is taken from AFM measurements ${ }^{15}$ ). The extremely low value of $x^{*}$ originating from this estimate is obviously in contradiction with the observed collectivity of the reorientation, and it has thus to be postulated that either $\tau^{*}$ is orders of magnitudes longer, $\sim 1 \mathrm{~s}$, than assumed above or that the excitation energy is distributed over $\sim 10^{9}$ molecules. With a large $\tau^{*}$, however, the electronic excitation energy would have to remain localized on the molecule for $\sim 1$ s, until nearly all molecules are electronically excited and rotational mobility is attained throughout the whole domain. This is in contradiction with the observation of reorientation processes on the time scale of nanoseconds. Therefore, these experiments supply additional evidence that not the electronic excitation provides rotational mobility, but its conversion to thermal energy, which is subsequently distributed over the aggregate. This yields a high density of thermally excited chromophores, $x$ 类 $\sim 1$, which drives photoreorientation. The time constant for the radiationless transitions that are responsible for the thermalization cannot be derived from $I_{\mathrm{th}}$, since it is related to the number of molecules thermally excited by each photon. The threshold intensity corresponds to a thermal energy of $0.7 \mathrm{eV} / \mathrm{s}$ absorbed by each molecule.

\section{Conclusions}

We have investigated the primary process of aminoazobenzene photoreorientation in LB films. The experimental results point to a mechanism of thermal dissipation of the electronic excitation energy, as postulated earlier. ${ }^{13}$ In deposited molecular films of compound $\mathbf{1}$ there is no detectable photostationary cis concentration, that would hint at trans $\rightarrow$ cis photoisomerization, which is the initial process for reorientation of azobenzenes in polymer or liquid-crystal matrices. The main difference in the molecular environment is a strong aggregation of the chromophores in the trans conformation that may restrict conformation changes, thus redirecting the deactivation of electronically excited states into alternate channels than the route via the cis isomer. We found experimental evidence that radiationless deactivation plays an important role, probably enabled by the coupling of excited states via a vibrational mode. This leads to the dissipation of electronic excitation into thermal energy which increases the rotational mobility of the chromophores.

We measured the intensity dependence of the reorientation efficiency. The results showed a pronounced collectivity of the photoreorientation process. A simple model describes this intensity dependence very well, and from this model, the magnitude $I_{\text {th }}$ of a characteristic intensity threshold can be extracted. From the analysis of these results, in particular the low value of $I_{\mathrm{th}}$, it is again obvious that the primary process of the photoreorientation cannot be located on an individual molecule. In contrast, the thermal energy delivered by a photon has to be distributed over the aggregate.

Acknowledgment. The authors would like to thank $\mathrm{H}$. Möhwald for valuable discussions and S. P. Palto (Moscow, Russia) for providing the azobenzene compound. Financial support from the Deutsche Forschungsgemeinschaft (Contract Lo352/3), the BMBF (Grant 03M4060) and the NATO under the Supramolecular Chemistry Programme (Contract SRG 930653) is gratefully acknowledged.

\section{References and Notes}

(1) Eich, M.; Wendorff, J. H.; Beck, J. H.; Ringsdorf, H. Macromol. Chem. Rapid Commun. 1987, 8, 59.

(2) Natansohn, A.; Rochon, P.; Gosselin, J.; Xie, S. Macromolecules 1992, 25, 2268

(3) Sekkat, Z.; Dumont, M. Appl. Phys. B 1992, 54, 486.

(4) Hvilstedt, S.; Andruzzi, F.; Ramanujam, P. S. Opt. Lett. 1992, 17, 1.

(5) Petri, A.; Kummer, S.; Anneser, H.; Feiner, F.; Bräuchle, C. Ber. Bunsen-Ges. Phys. Chem. 1993, 97, 308.

(6) Anneser, H.; Feiner, F.; Petri, A.; Bräuchle, C.; Leigeber, H.; Weitzel, H. D.; Kreuzer, F. H.; Haak, O.; Boldt, P. Adv. Mater. 1993, 5, 556.

(7) Dumont, M.; Sekkat, Z. SPIE J. 1992, 1774, 188.

(8) Ichimura, K.; Suzuki, Y.; Seki, T.; Kawanishi, Y.; Aoki, K. Langmuir 1988, 4, 5 .

(9) Kawanishi, Y.; Tamaki, T.; Sakuragi, M.; Seki, T.; Suzuki, Y. Langmuir 1992, 8, 2601.

(10) Möbius, G.; Pietsch, U.; Geue, T.; Stumpe, J.; Schuster, A.; Ringsdorf, H. Thin Solid Films 1994, 247, 235.

(11) Menzel, H.; Weichart, B.; Schmidt, A.; Paul, S.; Knoll, W.; Stumpe, J.; Fischer, T. Langmuir 1994, 10, 1926.

(12) Barnik, M. I.; Kozenkov, V. M.; Shtykov, N. M.; Palto, S. P.; Yudin, S. G. J. Mol. Electron. 1989, 5, 53.

(13) Palto, S. P.; Blinov, L. M.; Yudin, S. G.; Grewer, G.; Schönhoff, M.; Lösche, M. Chem. Phys. Lett. 1993, 202, 308.

(14) Schönhoff, M.; Grewer, G.; Palto, S. P.; Chi, L. F.; Fuchs, H.; Lösche, M. Thin Solid Films 1994, 242, 669.

(15) Schönhoff, M.; Chi, L. F.; Fuchs, H.; Lösche, M. Langmuir 1995, $11,163$.

(16) Palto, S. P.; Khavrichev, V. A.; Yudin, S. G.; Blinov, L. M.; Udal'yev, A. A. Mol. Mater. 1992, $2,63$.

(17) Barnik, M. I.; Palto, S. P.; Khavrichev, V. A.; Shtykov, N. M.; Yudin, S. G. Thin Solid Films 1989, 179, 493.

(18) Schönhoff, M. Ph.D. Thesis, University of Mainz, Mainz, 1994

(19) Wildes, P. D.; Pacifici, J. G.; Irick, G.; Whitten, D. G. J. Am. Chem. Soc. 1971, 93, 2004.

(20) Loucif-Saibi, R.; Nakatani, K.; Delaire, J. A.; Dumont, M.; Sekkat, Z. Chem. Mater. 1993, 5, 229.

(21) Barrett, C.; Natansohn, A.; Rochon, P. Macromolecules 1994, 27, 4781.

(22) Davydov, A. S. Theory of molecular excitons;; Plenum: New York, 1971.

(23) Liu, Z. F.; Morigaki, K.; Enomoto, T.; Hashimoto, K.; Fujishima, A. J. Phys. Chem. 1992, 96, 1875.

(24) Rau, H. In Photochemistry and Photophysics; Rabek, J. F., Eds.; CRC Press: Boca Raton, FL, 1990.

(25) Iwamoto, M.; Majima, Y.; Naruse, H. J. Appl. Phys. 1992, 72, 1631. (26) Majima, Y.; Kanai, Y.; Iwamoto, M. J. Appl. Phys. 1992, 72, 1637.

(27) Palto, S. P.; Malthete, J.; Germain, C.; Durand, G. Mol. Cryst. Liq. Cryst., submitted.

(28) Rau, H. J. Photochem. 1984, 26, 221.

(29) Palto, S. P.; Shtykov, N. M.; Khavrichev, V. A.; Yudin, S. G. Mol. Mater. 1992, $1,3$.

JP952052U 